\title{
Signature of non-isotropic distribution of stellar rotation inclination angles in the Praesepe cluster ${ }^{\star}$
}

\begin{abstract}
Geza Kovacs
Konkoly Observatory of the Hungarian Academy of Sciences, Konkoly Thege ut. 13-15, 1121 Budapest, Hungary

e-mail: kovacs@konkoly.hu

Received 12 June 2017 / Accepted 6 March 2018

ABSTRACT

The distribution of the stellar rotation axes of 113 main sequence stars in the open cluster Praesepe are examined by using current photometric rotation periods, spectroscopic rotation velocities, and estimated stellar radii. Three different samples of stellar rotation data on spotted stars from the Galactic field and two independent samples of planetary hosts are used as control samples to support the consistency of the analysis. Considering the high completeness of the Praesepe sample and the behavior of the control samples, we find that the main sequence $F-K$ stars in this cluster are susceptible to rotational axis alignment. Using a cone model, the most likely inclination angle is $76^{\circ} \pm 14^{\circ}$ with a half opening angle of $47^{\circ} \pm 24^{\circ}$. Non-isotropic distribution of the inclination angles is preferred over the isotropic distribution, except if the rotation velocities used in this work are systematically overestimated. We found no indication of this being the case on the basis of the currently available data.
\end{abstract}

Key words. stars: rotation - open clusters and associations: individual: M 44

\section{Introduction}

Current space- and ground-based surveys have made a significant contribution to our understanding of stellar rotation (e.g., McQuillan et al. 2014; Delorme et al. 2011; Hartman et al. 2010). Because stellar rotation carries information on the angular momentum evolution of the stars themselves and on the whole cluster (but in a rather involved way), these data are also relevant for understanding the poorly known early phase of cluster evolution, including the loss of angular momentum, the importance of gas dynamics, turbulence, and the magnetic field (e.g., McKee \& Ostriker 2007).

Inspired by the recent finding of Corsaro et al. (2017) on the apparent large degree of rotational axis alignment in two old clusters, here we examine the distribution of the line-of-sight component of the orientation of the rotational axes of 113 main sequence stars in the $\sim 0.8$ Gyr old cluster Praesepe. To derive the inclination angles we use spectroscopic and photometric rotational data combined with stellar radii from stellar evolution models. The distribution of the inclination angles derived in this way are modeled by various assumptions on their underlying distributions.

\section{Stellar rotation data}

\subsection{Praesepe}

The dataset we used is based primarily on the availability of spectroscopically derived rotational velocities. The 152 stars

\footnotetext{
* Data are only available at the CDS, together with the other two compiled datasets used in this paper, via anonymous ftp to cdsarc.u-strasbg.fr (130.79.128.5) or via http://cdsarc.u-strasbg.fr/viz-bin/qcat?J/A+A/612/L2
}

listed in Mermilliod et al. (2009) with possible cluster membership have been cross-correlated with the 180 stars from the HATNet $^{1}$ survey Kovács et al. (2014) and with the 941 stars recently published by Rebull et al. (2017) from the Kepler twowheel (K2) survey. After various filter steps (e.g., lack of $v \sin i^{2}$, stars with multiple matches) we ended up with 120 stars that all have 2MASS (Skrutskie et al. 2006) and $V$ colors (nearly exclusively from APASS: Henden et al. 2016), spectroscopic and photometric rotational data (available at the CDS, together with the other two compiled datasets used in this paper). We note that this sample is unbiased. No cut was made on the basis of the value of $v \sin i$, period, and color. Furthermore, because of the high precision of the $\mathrm{K} 2$ observations, the period sample is nearly complete; the periodic signal detection rate is $86 \%$ for the 941 stars examined in the work of Rebull et al. 2017. The rotation periods are also highly reliable, with an overall relative error of $3 \%$, based on the comparison of the common stars in the Kovács et al. 2014 and Rebull et al. 2017 samples, separated by several years.

To estimate the inclination angle, we needed to derive stellar radii. To achieve this goal, we opted to use the IRFM-based $T_{\text {eff }}$ calibration of Huang et al. (2015) from Johnson/2MASS $V-J, V-K$ colors. The observed magnitudes were dereddened by assuming $E(B-V)=0.027$ and standard extinction law (see Taylor 2006; Yuan 2013). By considering the different reddenings used, our temperatures are consistent with those given by Cummings et al. (2017) in their chemical abundance analysis of the same cluster. These $T_{\text {eff }}$ values were matched with the $Z=0.0200$, age $=0.8 \mathrm{Gyr}$ PARSEC isochrone of Marigo et al. (2017). With $Z_{\odot}=0.0152$ used in the PARSEC models, the adopted metal abundance is consistent with those given in

2 Throughout the paper we use the shorthand notation $v \sin i$ and $\sin i$, respectively, for the spectroscopic rotational velocity $V_{\mathrm{eq}} \sin (i)$ (with $V_{\mathrm{eq}}$ for the equatorial velocity) and for the sine of the inclination angle.
} 


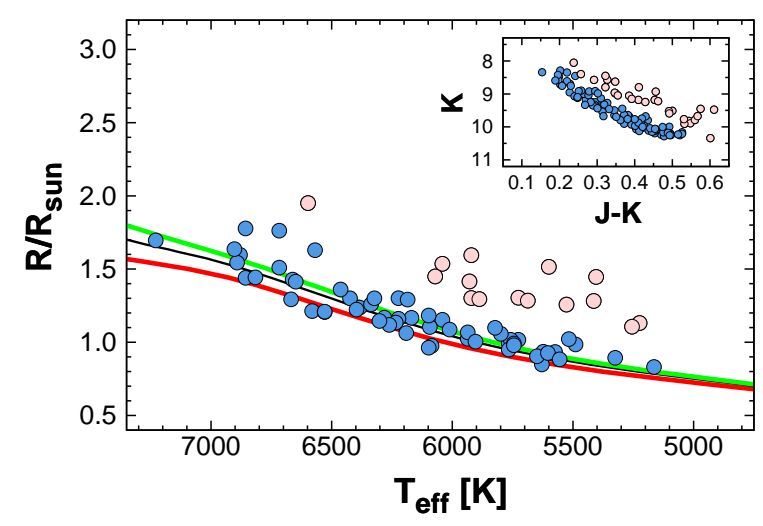

Fig. 1. Comparison of the radii derived from interferometrically calibrated photometric data by Bourgés et al. (2017) with the $0.8 \mathrm{Gyr}$ PARSEC isochrones for $[\mathrm{Fe} / \mathrm{H}]=0.0,+0.12$, and +0.19 , plotted with red, black, and green lines, respectively. The outliers are indicated by the pale dots and constitute a subset of the CMD outliers shown in the inset.

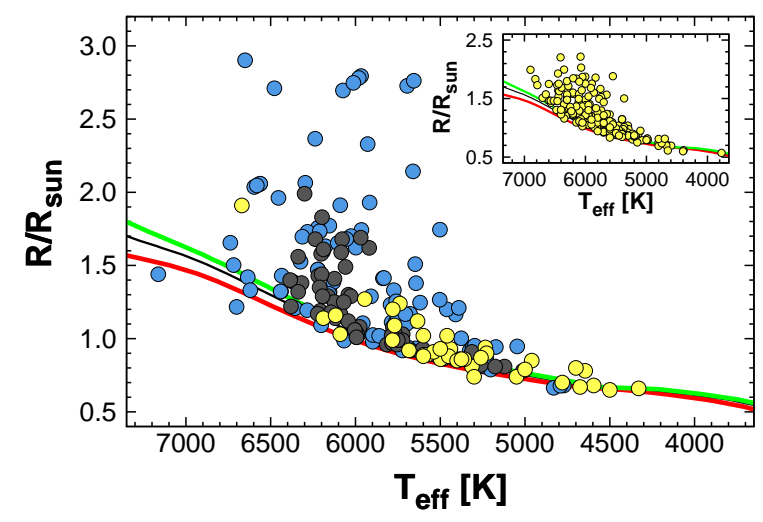

Fig. 2. Comparison of the radii based on the PARSEC stellar evolutionary models for Praesepe with other stellar radii derived for Galactic field stars. The isochrones shown in Fig. 1 are used. The interferometric radii collected by Boyajian et al. (2013) are shown by blue dots, those derived from the extrasolar planetary systems in the Kepler field (Winn et al. 2017) by deep gray dots, and hot Jupiter hosts elsewhere (Maxted et al. 2015, extended/updated for this study) by yellow dots. The inset shows the same kind of plot, but using the large compilation of transiting planet hosts by Hartman et al. (2016).

current spectroscopic studies (i.e., Boesgaard et al. 2013; see also Cummings et al. 2017). The age used is in agreement with the value currently predicted by models including the effect of stellar rotation (Brandt \& Huang 2015), but older than the standard age of $\sim 0.6$ Gyr (e.g., Fossati et al. 2008). Because the stars studied in this work are far from the turn-off luminosity of the cluster, this difference has no effect whatsoever on the results presented in this paper. Once the observed and isochrone temperatures are matched, the radii are estimated from the model luminosities, using the blackbody formula.

To check the consistency between the radii determined above and those derived by various other methods, first we took the database of (Bourgés et al. 2014, 2017) containing photometrically calibrated stellar angular diameters from direct interferometric data. We found 74 matches with our Praesepe sample. Assuming a cluster distance of 182 pc (e.g., van Leeuwen 2009), the angular diameter $\phi$ was converted to stellar diameter by using the formula $R / R_{\odot}=19.56 \phi$ [mas]. These radii are plotted in Fig. 1 as a function of $T_{\text {eff }}$. We see that the test dataset is rather sensitive to blending. Therefore, we think that the overall match of the isochrones with the part between the lower envelope and the ridge supports the radii values used in the present work, based on the $[\mathrm{Fe} / \mathrm{H}]=+0.12$ isochrone.

In the second consistency check (Fig. 2) we performed a non-direct comparison by taking the interferometric data on 125 Galactic field stars published by Boyajian et al. (2013). In addition to these data we added the planetary host samples of hot Jupiters (HJs) and Kepler planets (KOIs) from Table 1 and the compilation of 199 planetary systems by Hartman et al. (2016). Again, with the obviously older age and wide metallicity spread of these field star data, the isochrone used in this work for Praesepe (representing a relatively young and moderately metal-rich sample) seems to be well justified.

\subsection{Control samples}

We used five control samples to gain further support for the better assessment of the statistical significance of the rotation angle distribution derived for Praesepe. These samples (see Table 1) were chosen under the assumption of near homogeneity of rotation axis distributions within each set. Because of the observed transits and the likely alignment of the stellar rotation and orbital angular momentum vectors, the two planet host samples are expected to show dominance of inclination angles close to $90^{\circ}$ (exceptions are the few non-aligned systems; see Winn et al. 2017). On the other hand, the magnetic, chemically peculiar (MCP) stars of Field (A) and (B) are expected to have random rotational axis orientations, and therefore may serve as good control groups for testing the isotropic distribution. The same is true for the spotted star sample Field (C), but they are members of the Kepler field, and so there is basically no selection effect for the amplitude of the observable photometric variation. Therefore, objects with small inclination angles have an equal probability of being included in the sample; however, the limited accuracy of $v \sin i$ could still pose some constraints on slow rotators.

Two selection criteria are applied to all items used in the analysis: i) avoiding poorly determined periods, stars with $P_{\text {rot }}>40$ days are omitted, and ii) avoiding clearly bad items, stars with $\sin i>2.0$ are omitted. Except for Field (B), where the stellar radii are calculated from the tabulated luminosity and effective temperature, we used the radii given in the respective publications. For Field (B) there are 1347 stars in the catalog of Netopil et al. (2017), but only 193 stars have all the necessary parameters for this study. The Field (C) data resulted from the cross-correlation of several spectroscopic and photometric catalogs covering the Kepler field (see Table 1 for the respective publications). Radii for this dataset are as given in the corresponding catalogs or were taken from Huber et al. (2014) when needed.

\section{Method of analysis}

In Sect. 4 we first make a simple comparison of the cumulative distribution functions (CDFs) for $\sin i$ derived from the observed $v \sin i$, rotational period $P_{\text {rot }}$, and estimated stellar radius $R_{\text {star }}$. With the assumption of dominating equatorial spots in the derived rotational periods, we use the formula $\sin i=v \sin i P_{\text {rot }} /\left(2 \pi R_{\text {star }}\right)$ to estimate $\sin i$. Second, after a brief classification of the CDFs, following Jackson et al. (2010), we model the observed distributions by assuming various distributions of $i$ and considering the distortion caused by the observational errors on these theoretical distributions. From the formula already quoted, we have

$\sin i_{\mathrm{obs}}=\frac{\left(1+\xi_{\mathrm{P}} / P_{\mathrm{rot}}^{0}\right)\left(1+\xi_{\mathrm{v} \sin \mathrm{i}} / v \sin i^{0}\right)}{1+\xi_{\mathrm{R}} / R_{\mathrm{star}}^{0}} \sin i$. 
Table 1. Summary of the datasets used in this paper

\begin{tabular}{lcccr}
\hline \hline Dataset & $N_{\text {tot }} / N$ & $\langle v \sin i\rangle$ & $\langle\sigma(v \sin i)\rangle$ & Source \\
\hline Praesepe & $120 / 113$ & 8.4 & 1.5 & M09, K14, R17 \\
HJ $_{\text {host }}$ & $39 / 39$ & 4.0 & 0.6 & M15 \\
KOI $_{\text {host }}$ & $61 / 61$ & 6.2 & 1.1 & W17 \\
Field (A) & $97 / 91$ & 51.3 & 20.0 & A01 \\
Field (B) & $193 / 179$ & 46.1 & 14.8 & Net17 \\
Field (C) & $55 / 46$ & 24.8 & 1.6 & M13, N15, N17 \\
& & & & N13, R15, Mc14
\end{tabular}

Notes. $\mathrm{HJ}_{\text {host }}$ and $\mathrm{KOI}_{\text {host }}$ stand for the two separate sets of extrasolar planet host stars (mostly with hot Jupiters and various Kepler planets, respectively). Field $(\mathrm{A}, \mathrm{B}, \mathrm{C})$ stand for three sets of Galactic field stars. $\langle v \sin i\rangle$ and $\langle\sigma(v \sin i)\rangle$, respectively, denote the averages of the $v \sin i$ values and the averages of their errors given in the source for $v \sin i$. The total number of items and the number actually used in this work are denoted by $N_{\text {tot }}$ and $N$, respectively. See Sect. 2.2 for the description of the selection criteria.

References. M09: Mermilliod et al. (2009); K14: Kovács et al. (2014); R17: Rebull et al. (2017); M15: Maxted et al. (2015) + current literature (11 stars); W17: Winn et al. (2017); A01: Abt (2001); Net17: Netopil et al. (2017); M13: Molenda-Zakowicz et al. (2013); N15: Niemczura et al. (2015); N17: Niemczura et al. (2017); N13: Nielsen et al. (2013); R15: Reinhold \& Gizon (2015); Mc14: McQuillan et al. (2014).

Here $P_{\text {rot }}^{0}, R_{\mathrm{star}}^{0}$, and $v \sin i^{0}$ denote the true stellar parameters, which we approximate with their observed/computed counterparts. The errors $\xi_{\ldots}$ are Gaussian, except for $\xi_{v \sin i}$, which can be nicely represented by a LogNormal distribution. The noise parameters are taken from the observations (see below).

In modeling the observed CDF with a given distribution of $\sin i$, we use Eq. (1) with 50 different realizations of $\left(\sin i, \xi_{\mathrm{R}}, \xi_{\mathrm{P}}, \xi_{v \sin i}\right)$ for all members of the sample. The CDFs of these $\sin i_{\text {obs }}$ values are computed and an average CDF is obtained with the corresponding standard deviations derived from the 50 realizations. The quality of the fit is parameterized by the RMS of the residuals between the target and the average CDF.

Choosing the size of the error components in the modeling is important in general, and could be crucial in the interpretation of the resulting underlying distribution of $\sin i$. This is especially true for $\xi_{v \sin i}$. After an exhaustive series of tests, we decided to trust in the published errors and use them without any up- or down-scaling, depending on some consistency criteria that might be applied to the given dataset (e.g., using unreasonable down-scaling to reach better agreement with the expected aligned distribution for the planetary hosts). For the period and radius, the sources of the datasets include irregular noise information. Therefore, we decided to estimate the expected errors. Based on the comparison of the periods of Praesepe from Kovács et al. (2014) and Rebull et al. (2017), we found that the period errors are proportional to the period with a scaling factor of 0.03 . For the radius errors we use the publication of Winn et al. (2017). The radius errors given in this publication show a reasonably tight correlation with the radii. In summary, assuming standard Gaussian distributions, we use the following error formulae

$\xi_{\mathrm{P}}=\mathrm{GAUSS}_{\mathrm{P}} \times 0.03 P_{\mathrm{rot}}^{0}$,

$\xi_{\mathrm{R}}=\mathrm{GAUSS}_{\mathrm{R}} \times\left|0.27 R_{\mathrm{star}}^{0}-0.2\right|$.

We note that the results presented in this paper are only mildly sensitive to the assumed noise in the period and radius. The errors on $v \sin i$ are far more important.
Table 2. Contingency table for the K-S statistics of the CDFs of the observed $\sin i$

\begin{tabular}{|c|c|c|c|c|c|c|}
\hline Dataset & Praesepe & $\mathrm{HJ}_{\text {host }}$ & $\mathrm{KOI}_{\text {host }}$ & Field (A) & Field (B) & Field (C) \\
\hline Praesepe & 1.000 & 0.103 & 0.005 & 0.018 & 0.002 & 0.245 \\
\hline $\mathrm{HJ}_{\text {host }}$ & 0.103 & 1.000 & 0.028 & 0.000 & 0.000 & 0.005 \\
\hline $\mathrm{KOI}_{\text {host }}$ & 0.005 & 0.028 & 1.000 & 0.000 & 0.000 & 0.000 \\
\hline Field (A) & 0.018 & 0.000 & 0.000 & 1.000 & 0.374 & 0.620 \\
\hline Field (B) & 0.002 & 0.000 & 0.000 & 0.374 & 1.000 & 0.410 \\
\hline Field (C) & 0.245 & 0.005 & 0.000 & 0.620 & 0.410 & 1.000 \\
\hline
\end{tabular}

Notes. The entries show the probabilities $P=\operatorname{Pr}\left[d_{\max }>d_{\max }^{\text {obs }}\right]$, where $d_{\max }^{\text {obs }}$ is the observed maximum difference between the CDFs of the pair of datasets tested. Low probabilities indicate that the two CDFs are drawn from different distributions. For easier comparison, the $P>0.05$ cases are in bold. We use two-sample Kolmogorov-Smirnov statistics.

Finally, the inclination angles are generated by following either the isotropic (Chandrasekhar \& Münch 1950, see also Curé et al. 2014) or a cone distribution (see Jackson et al. 2010). While the isotropic model has no free parameters except for the generally accepted noise model for the dataset, the cone distribution has two adjustable parameters: $\alpha$, the inclination of the axis of the cone, and $\lambda$, half of the opening angle of the cone. Best fit cone parameters are searched for by a simple grid search in $[0, \pi / 2]$ for both parameters. The errors on these parameters come from the possible parameter regimes allowed by the $1 \sigma$ scatter of the realization-dependent standard deviations of the residuals between the target $\mathrm{CDF}$ and the best fitting model. In the course of the error estimation, using the inverse of the $\mathrm{CDF}$ residuals as weights, the cone parameters are updated.

\section{Results}

First we compare the distributions of the $\sin i$ values derived from the observations for the six datasets. Figure 3 shows the result of this comparison, clearly indicating the expected similarity of the various groups of stars. To make this statement more quantitative, we perform a two-sample Kolmogorov-Smirnov (K-S) test for the pairs of the datasets. As expected, Table 2 confirms the classification suggested by Fig. 3. Although the relative errors of $v \sin i$ vary by several factors over the samples, there are datasets of similar quality that show markedly different distributions, for example Praesepe and the planet hosts, and similar distributions with significantly different noise properties, for example set $(C)$ in the field star sample ${ }^{3}$. Therefore, the differences in the distributions likely reflect real differences in the distributions of the inclination angles.

To model the underlying distribution of $\sin i$ we followed the methodology described in Sect. 3. Figure 4 shows the result for the six datasets studied in this work. Starting with Praesepe, we see that the isotropic distribution is clearly distinct from the observed distribution ${ }^{4}$. However, the shape of

\footnotetext{
3 The relatively large $\mathrm{K}-\mathrm{S}$ probability associated with the Praesepe Field (C) samples is attributed in part to the small sample size of the latter.

4 In testing the robustness of this result against binaries and blends, we omitted the $28 \mathrm{CMD}$ outliers shown in Fig. 1 and found that the distinctiveness of the two distributions remains, albeit with a lower significance: the RMS between the two CDFs decreased from 0.076 (full sample) to 0.062 (without the CMD outliers). On the other hand, omitting only the 31 spectroscopic binaries - half of them are not outliers leads to an increase in the RMS to 0.095 .
} 


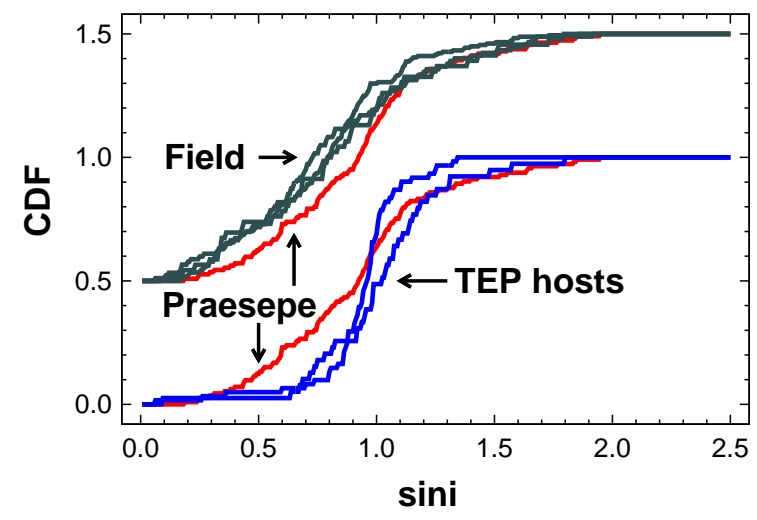

Fig. 3. Comparison of the CDFs of sin i obtained from the observed $v \sin i, P_{\text {rot }}$, and estimated $R_{\text {star }}$ values. For better visibility, the CDFs are shifted vertically by 0.5 with respect to those of the planetary hosts (TEPs). The three gray lines correspond to the three sets of Galactic field stars given in Table 1. Similarly, the two blue lines are related to the exoplanet host stars. The CDF for Praesepe is shown twice for easier comparison with the CDFs corresponding to the different datasets.

the CDF is very similar to that of the observed CDF. This prompted us to investigate the possibility of the overestimation of the $v \sin i$ values. The Mermilliod et al. (2009) data come from the long-term monitoring of this cluster by the CORAVEL instrument. The correlation profiles were modeled by Gaussians, assuming broadening factors from rotation, stellar turbulence, and instrumental effects (see Benz \& Mayor 1984). From the $\sigma$ of the fitted profile, $v \sin i$ is computed from $v \sin i=A \sqrt{\sigma^{2}-\sigma_{0}^{2}}$. Here $A=1.9$ and $\sigma_{0}$ stands for the broadening of the non-rotating star. We find that a relatively small increase in $\sigma_{0}$ of $0.2 \mathrm{kms}^{-1}$ makes the isotropic model valid. Although this is still considerably far from the expected range of $\sigma_{0}$ (see Fig. 1 in Queloz et al. 1998), it calls for even higher precision data to give a more reliable answer on the issue of zero point of the $v \sin i$ data. On the other hand, by checking other (even though sparse and, in general, less accurate) data sources on Praesepe (Cummings et al. 2017; Quinn et al. 2012; Pace \& Pasquini 2004; Boesgaard et al. 2004; Rachford 1998; Malavolta et al. 2016; Mann et al. 2017) we find that most of the published values $(\sim 80 \%)$ are an average of $10-20 \%$ higher than our values. Therefore, we think that the current data lend support to a non-isotropic inclination angle distribution rather than to the isotropic one.

For the other datasets we see a comfortable overall consistency between the expected distributions and the best fitting models. The optimal cone models (with large parameter scatter, as expected) are indistinguishable from the isotropic model for all field star samples, although there is a slight indication for the underestimation of the observational noise for Field (C): the observed CDF is shallower than that of the isotropic distribution. The opposite is likely to be true for the planetary host samples, especially for the KOI sample. We find that decreasing the noise on $v \sin i$ by a factor of two (relative to the values given by Winn et al. 2017), the fit improves substantially with $\sigma_{\text {cone }}=0.028 \pm 0.008$ and cone parameters $\alpha=80^{\circ} \pm 11^{\circ}$ and $\lambda=27^{\circ} \pm 18^{\circ}$. These cone parameters may imply the presence of some oblique systems in the KOI sample. Instead of making any far-reaching conclusion of this sort from this test alone, we refer to Hirano et al. (2014) and Winn et al. (2017) for further discussion of the topic. However, we draw attention to the importance
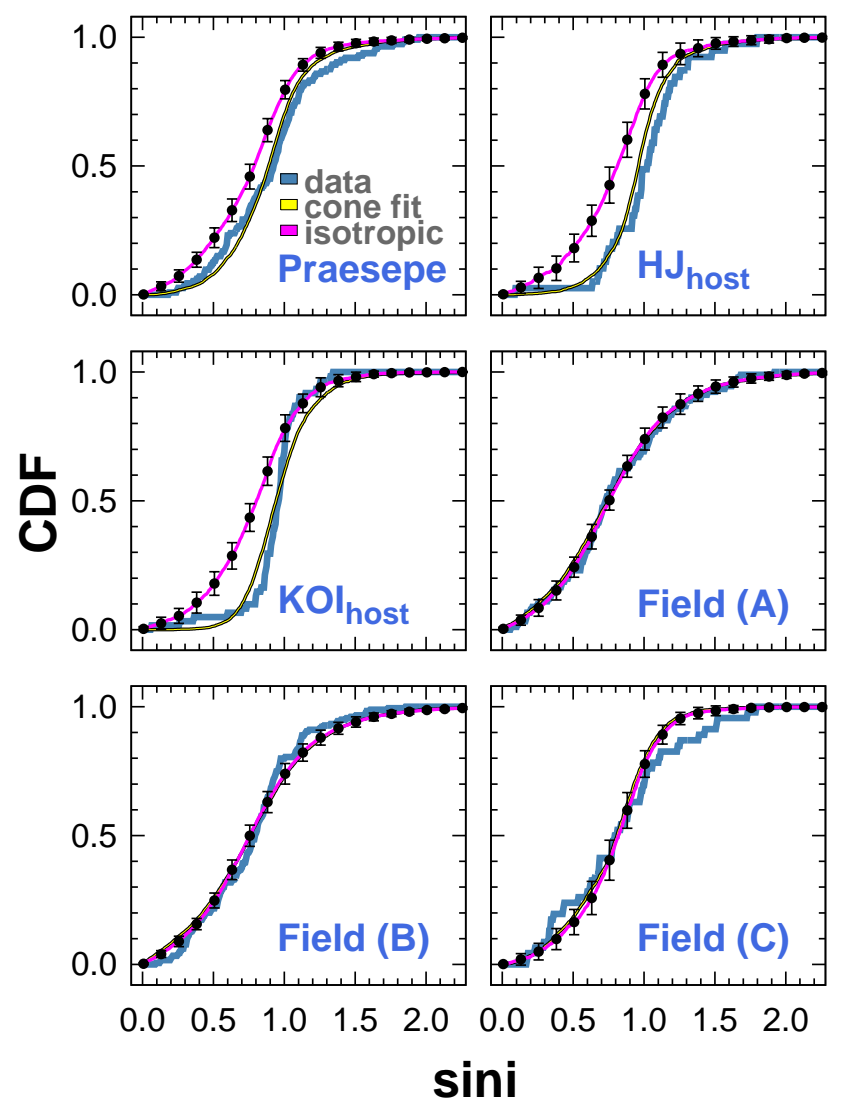

Fig. 4. Observed cumulative distributions of $\sin i$ and the associated model distributions assuming isotropic and cone distributions (see Jackson et al. 2010) for the inclination angle. See Table 3 for the $(\alpha, \lambda)$ parameters used in the cone distributions. The error bars correspond to $1 \sigma$ limits derived from the random simulations of the theoretical CDFs. To avoid crowding, we show them only for the isotropic distributions because the errors for the cone distributions are very similar.

of proper noise estimation when modeling the distribution of the inclination angles from spectroscopic rotation velocities.

\section{Conclusions}

By using recently determined stellar rotation periods, available spectroscopic rotation velocities, and evolutionary stellar radii based on IRFM-calibrated effective temperatures, we derived the distribution of the line-of-sight inclination angles from 113 main sequence $F-K$ stars in the Praesepe open cluster. With the aid of statistical modeling, we found signatures of a broad rotational axis alignment. The cone model of Jackson et al. (2010) yielded an overall cone axis angle of $76^{\circ} \pm 14^{\circ}$ and an opening angle of $47^{\circ} \pm 24^{\circ}$. Isotropic angle distribution is far less likely, except when the spectroscopic rotational velocities used in this work are systematically overestimated. Although we cannot exclude this from being the case, a comparison made with other spectroscopic data available in the literature makes this possibility less likely.

This is the first result on a possible alignment of stellar rotation axes on a large sample. In addition, the sample used in the present work is highly complete with regard to the available vsin $i$ data. Employing only about 40 stars per cluster, earlier investigations by Jackson et al. (2010) concluded with the low likelihood of alignment in the Pleiades and Alpha Per clusters (see also 
Table 3. Summary of the CDF model matches

\begin{tabular}{lcccc}
\hline \hline Dataset & $\alpha$ & $\lambda$ & $\sigma_{\text {cone }}$ & $\sigma_{\text {iso }}$ \\
\hline Praesepe & $76 \pm 14$ & $47 \pm 24$ & $0.033 \pm 0.011$ & $0.079 \pm 0.017$ \\
HJ $_{\text {host }}$ & $85 \pm 09$ & $18 \pm 13$ & $0.034 \pm 0.015$ & $0.142 \pm 0.024$ \\
KOI $_{\text {host }}$ & $81 \pm 14$ & $24 \pm 23$ & $0.049 \pm 0.011$ & $0.128 \pm 0.018$ \\
Field (A) & $52 \pm 43$ & $76 \pm 46$ & $0.015 \pm 0.009$ & $0.016 \pm 0.008$ \\
Field (B) & $55 \pm 46$ & $69 \pm 58$ & $0.025 \pm 0.007$ & $0.031 \pm 0.009$ \\
Field (C) & $48 \pm 42$ & $75 \pm 32$ & $0.046 \pm 0.013$ & $0.052 \pm 0.013$
\end{tabular}

Notes. The cone model parameters $\alpha$ and $\lambda$ denote the cone axis inclination angle and half of the opening angle, respectively. The standard deviations of the residuals between the model and the target CDFs are denoted by $\sigma_{\text {cone }}$ and $\sigma_{\text {iso }}$ for the cone and isotropic distribution, respectively. Errors have been computed from the Monte Carlo simulation, as described at the end of Sect. 3, and are subject to realization dependence at the $\sim 10 \%$ level.

the recent analysis by Jackson et al. 2018). To date, the only work suggesting stellar spin axis alignment in clusters is that of Corsaro et al. (2017). However, their result is based on small samples (25 stars for NGC 6791 and 23 stars for NGC 6819).

The currently available data on Praesepe allow only a rather inaccurate estimation of the inclination angles. With the type of instrumentation used in the field of extrasolar planets, the same accuracy of $\sim 0.5 \mathrm{~km} \mathrm{~s}^{-1}$ in $v \sin i$ could be reached. With a further improvement in the photometry and blend analysis, the error of the radius estimates could also be decreased to $3-5 \%$; with the already accurate rotation periods, this would enable us to estimate $\sin i$ with an accuracy of $\sim 7-10 \%$. This is a two- to threefold decrease in error compared with what we have now.

Our findings in this work and those of Corsaro et al. (2017) are very difficult to understand by current stellar formation and cluster evolution theories. It is hard to see how some complicated magnetohydrodynamic effects can lead to even broadly organized spin axis distribution on a gigayear timescale after the star formation period.

At this moment we think that the most effective way to make progress in the more secure disentangling of the various types of distribution is to acquire more accurate spectroscopic rotational velocities as described above. This, together with a better estimation of the noise budget, will most likely lead to a cleaner observational input for the theory of rotational axis alignment.

Acknowledgements. The thorough reports of the referee, leading to a deep revision of the methodology used and the conclusions drawn, are greatly appreciated We would like to thank Luisa Rebull for the prompt and instructive response to our inquiry about data availability before journal publication. Martin Netopil is acknowledged for his clarification on the error budget of the rotational velocities of mCP stars in their database. We thank Stephane Udry for his help in understanding the assignment of errors to the CORAVEL rotational velocities. This research has made use of the VizieR catalogue access tool, CDS, Strasbourg, France.

\section{References}

Abt, H. A. 2001, ApJ, 122, 2008

Benz, W., \& Mayor, M. 1984, A\&A, 138, 2183

Boesgaard, A. M., Armengaud, E., \& King, J. R. 2004, ApJ, 605, 864

Boesgaard, A. M., Roper, B. W., \& Lum, M. G. 2013, ApJ, 775, 58

Bourgés, L., Lafrasse, S., Mella, G., et al. 2014, ASP Conf. Ser., 485, 223

Bourgés, L., Mella, G., Lafrasse, S., et al. 2017 VizieR Online Data Catalog: II/346

Boyajian, T. S., von Braun, K., van Belle, G., et al. 2013, ApJ, 771, 40

Brandt, T. D., \& Huang, C. X. 2015, ApJ, 807, 24

Chandrasekhar, S., \& Münch, G. 1950, ApJ, 111, 142

Corsaro, E., Lee, Y-N., Garcia, R. A., et al. 2017, Nat. Astron., 1, 64

Cummings, J. D., Deliyannis, C. P., Maderak, R. M., Steinhauer, A. 2017, AJ, 153,128

Curé, M., Rial, D. F., Christen A., Cassetti, J. 2014, A\&A, 565, A85

Delorme P., Collier Cameron A., Hebb L., et al. 2011, MNRAS, 413, 2218

Fossati, L., Bagnulo, S., Landstreet, J., et al. 2008, A\&A, 483, 891

Hartman, J. D., Bakos, G. A., Kovács, G., \& Noyes, R. W. 2010, MNRAS, 408, 475

Hartman, J. D., Bakos, G. Á., Bhatti, W., et al. 2016, AJ, 152, 182

Henden, A. A., Templeton, M., Terrell, D., et al. 2016, VizieR Online Data Catalog: II/336

Hirano, T., Sanchis-Ojeda, R., Takeda, Y., et al. 2014, ApJ, 783, 9

Huang, Y., Liu, X.-W., Yuan, H.-B., et al. 2015, MNRAS, 454, 2863

Huber, D., Aguirre, V. S., Matthews, J. M., et al. 2014, ApJS, 211, 2

Jackson, R. J., \& Jeffries, R. D. 2010, MNRAS, 402, 1380

Jackson, R. J., Deliyannis, C. P., \& Jeffries, R. D. 2018, MNRAS, 476, 3245

Kovács, G., Hartman, J. D., Bakos, G. Á. et al. 2014, MNRAS, 442, 2081

Malavolta, L., Nascimbeni, V., Piotto, G., et al. 2016, A\&A, 588, 118

Mann, A. W., Gaidos, E., Vanderburg, A., et al. 2017, ApJ, 153, 64

Marigo, P., Girardi, L., Bressan, A., et al. 2017, ApJ, 835, 77

Maxted, P. F. L., Serenelli, A. M., \& Southworth, J. 2015, A\&A, 577, 90

McKee, C. F., \& Ostriker, E. C. 2007, ARA\&A, 45, 565

McQuillan, A., Mazeh, T., \& Aigrain S. 2014, ApJS, 211, 24

Mermilliod J.-C., Mayor M., Udry S. 2009, A\&A, 498, 949

Molenda-Zakowicz, J., Sousa, S. G., Frasca, A., et al. 2013, MNRAS, 434, 1422

Netopil, M., Paunzen, E., Hummerich, S., \& Bernhard, K. 2017, MNRAS, 468, 2745

Nielsen, M. B., Gizon, L., Schunker, H., \& Karoff, C. 2013, A\&A, 557, L10

Niemczura, E., Murphy, S. J., Smalley, B. et al. 2015, MNRAS, 450, 2764

Niemczura, E., Polinska, M., Murphy, S. J., et al. 2017, MNRAS, 470, 2870

Pace, G., \& Pasquini, L. 2004, A\&A, 426, 1021

Queloz, D., Allain, S., Mermilliod, J.-C., Bouvier, J., \& Mayor, M. 1998, A\&A, 335,183

Quinn, S. N., White, R. J., Latham, D. W., et al. 2012, ApJ, 756, L33

Rachford, B. L. 1998, ApJ, 505, 255

Rebull, L. M., Stauffer, J. R., Hillenbrand, L. A., et al. 2017, ApJ, 839, 92

Reinhold, T., \& Gizon, L. 2015, A\&A, 583, A65

Skrutskie, M. F., Cutri, R. M., R. Stiening, R., et al. 2006, AJ, 131, 1163

Taylor, B. J., 2006, AJ, 132, 2453

Yuan, H. B., Liu, X. W., \& Xiang, M. S. 2013, MNRAS, 430, 2188

van Leeuwen, F. 2009, A\&A, 497, 209

Winn, J. N., Petigura, E. A., Morton, T. D., et al. 2017, AJ, 154, 270 